\title{
Erratum to: A new view of radiation-induced cancer: integrating short- and long-term processes. Parts I and II
}

\author{
Igor Shuryak · Philip Hahnfeldt • Lynn Hlatky • \\ Rainer K. Sachs $\cdot$ David J. Brenner
}

Published online: 24 August 2011

(C) Springer-Verlag 2011

\section{Erratum to: Radiat Environ Biophys (2009) 48:263-274 DOI 10.1007/s00411-009-0230-3; Radiat Environ Biophys (2009) 48:275-286 DOI 10.1007/s00411-009-0231-2}

We wish to correct some minor errors in A New View of Radiation-Induced Cancer: Integrating Short- and LongTerm Processes, Parts I and II, which appeared in Radiation and Environmental Biophysics (Shuryak et al. 2009a, b). The Results and Figures remain unchanged. Likewise, the conclusion of the two papers, that this mechanistically based formalism provides a practical methodology for predicting radiation-induced cancer risks at radiotherapeutic doses, still stands.

The online version of the original articles can be found under doi:10.1007/s00411-009-0230-3; 10.1007/s00411-009-0231-2.

Electronic supplementary material The online version of this article (doi:10.1007/s00411-011-0378-5) contains supplementary material, which is available to authorized users.

I. Shuryak · D. J. Brenner $(\bowtie)$

Center for Radiological Research,

Columbia University Medical Center,

630 West 168th St., New York, NY 10032, USA

e-mail: djb3@columbia.edu

I. Shuryak

e-mail: ishuryak@gmail.com

P. Hahnfeldt · L. Hlatky

Caritas St. Elizabeth's Medical Center,

Tufts University School of Medicine, Boston, MA, USA

R. K. Sachs

Departments of Mathematics and Physics,

University of California Berkeley, Berkeley, CA, USA
1. There was an error in Eq. (22) of the Appendix of Shuryak et al. (2009a), which we would like to correct. Details are given here and in the Revised Appendix (Supplementary Material for this Erratum):

The Appendix in Shuryak et al. (2009a) derived the probability, $F(k)$, that a cell initiated by a $k$ th radiation dose fraction produces a clone that survives all subsequent dose fractions. The incorrect equation for this quantity appeared as Eq. (22) of the Appendix and was repeated on page 269 of Shuryak et al. (2009a). In both the locations, it should be replaced with the following formula:

$$
\begin{aligned}
& F(k)=\frac{1}{\Delta}, \quad \text { where } \Delta=1+\sum_{j=k+1}^{K}\left[\frac{S^{k-j}(1-S)}{\prod_{i=k}^{j-1} R(i)}\right], \\
& S=\exp \left[-\alpha d-\beta d^{2}\right], \text { and } R(i)=\frac{n^{-}(i+1)}{n^{+}(i)}
\end{aligned}
$$

This corrected formula is explicitly derived and interpreted in the Revised Appendix (Supplementary Material for this Erratum).

Applying the correct equation resulted in small corrections to the best-fit values of the carrying capacity for the number of pre-malignant cells per niche. This carrying capacity, $Z$, represents the homeostatically regulated number of pre-malignant cells per niche. Both the published values of $Z$ (from Table 2 of Shuryak et al. 2009b) and the corrected values of $Z$ are given in the table below. All other parameter estimates and results are unchanged.

2. We clarify that the quantity that we calculate, the hazard function for new malignant cells, is in fact more accurately described by Eq. (1) of Shuryak et al. (2009a). The hazard function approximation used in 
the calculations, Eq. (2), produces only very slightly different results, and only when the cancer risk is high.

3. Both after Eq. (9) and before Eq. (11) of Shuryak et al. (2009a), the average number of pre-malignant clones should be described as proportional to $a X I(k)$, rather than equal to $a X I(k)$.

4. Two curly brackets were missing from the numerator of Eq. (14) of Shuryak et al. (2009a), which describes the absolute radiation-induced cancer risk as a function of age at exposure and time since exposure. The corrected equation is given here:

$$
\begin{aligned}
& A_{\mathrm{rad}}\left(T_{x}, T_{y}\right) \\
& \quad=\left[N_{\mathrm{rad}} E\left(T_{x}, T_{y}\right) C_{\mathrm{rad}}\left(T_{x}, T_{y}\right)+N_{\mathrm{rad}} N\left(T_{x}, T_{y}\right)\right] P\left(T_{x}, T_{y}\right) \\
& \quad=\frac{a}{b}\left[\frac{\left\{\left(\exp \left[b T_{x}\right]-1\right) S f(Z, D)+b X I S f(D) \exp \left[b T_{y}\right]\right\}(1+Y D)}{1+Y D\left(1-\exp \left[-\delta T_{y}\right]\right)}\right. \\
& \left.\quad+\exp \left[b T_{y}\right]-1\right] \exp \left[-c\left(T_{x}+T_{y}\right)^{2}\right]
\end{aligned}
$$

Table Correction to Table 2 of Shuryak et al. (2009b)

\begin{tabular}{lll}
\hline Cancer & $\begin{array}{l}\log _{10} Z \text { in Shuryak } \\
\text { et al. }(2009 b)\end{array}$ & $\log _{10} Z$ corrected \\
\hline Stomach & $3.58(2.65,3.66)$ & $3.99(3.07,4.22)$ \\
Lung & $4.99(3.80,5.56)$ & $5.61(3.04,5.97)$ \\
Colon & $0.301(0.00,0.628)$ & $0.760(0.442,1.12)$ \\
Rectum & $2.09(0.978,3.05)$ & $2.40(2.27,3.55)$ \\
Pancreas & $3.23(2.73,4.09)$ & $4.16(3.70,5.12)$ \\
Bladder & $1.96(1.72,2.13)$ & $2.78(2.51,2.94)$ \\
Breast & $6.65(5.47, \infty)$ & $7.00(5.83, \infty)$ \\
CNS & $5.69(4.27, \infty)$ & $6.72(5.16, \infty)$ \\
Thyroid & $4.03(3.02,6.15)$ & $4.26(3.11,6.37)$ \\
\hline
\end{tabular}

Various parameter values were adjusted for best fits to the data. The only parameter affected by the error in Eq. (22) was the carrying capacity, $Z$, whose best fits were changed as shown here, with $95 \%$ confidence limits in parentheses

\section{References}

Shuryak I, Hahnfeldt P, Hlatky L, Sachs RK, Brenner DJ (2009a) A new view of radiation-induced cancer: integrating short- and long-term processes. Part I: approach. Radiat Environ Biophys 48:263-274

Shuryak I, Hahnfeldt P, Hlatky L, Sachs RK, Brenner DJ (2009b) A new view of radiation-induced cancer: integrating short- and long-term processes. Part II: second cancer risk estimation. Radiat Environ Biophys 48:275-286 